\title{
The Copper-Catalyzed Asymmetric Allylic Substitution
}

\author{
Alexandre Alexakis*, Christophe Malan, Louise Lea, Karine Tissot-Croset, Damien Polet, and \\ Caroline Falciola
}

\begin{abstract}
This mini-review discusses the rapidly growing field of asymmetric copper-catalyzed chemistry. Although the allylic substitution has been less studied than the conjugate addition, recent breakthroughs have made this methodology a very valuable synthetic tool. Thus, a primary allylic halide or phosphate reacts with Grignard or diorganozinc reagents to afford the $S_{N}$, product (or $\gamma$-product) in high regio- and enantioselectivities. Besides the results of the authors, we present also other, different approaches to this reaction, with emphasis on the organometallic and the type of chiral ligand used.
\end{abstract}

Keywords: Asymmetry · Catalysis · Copper · Magnesium · Substitution · Zinc

\section{Introduction}

The allylic substitution reaction is one of the fundamental transformations in organic synthesis [1]. One of the key features of this reaction is its regioselectivity. Direct substitution at the carbon bearing the leaving group ( $\alpha$-attack) results in a formal $\mathrm{S}_{\mathrm{N}} 2$ substitution. Alternatively, the reaction may proceed by an $\mathrm{S}_{\mathrm{N}}$ ' mode ( $\gamma$-attack) with concomitant displacement of the double bond (Scheme 1).

When a $\mathrm{C}-\mathrm{C}$ bond formation is carried out with a carbon nucleophile, it is usually promoted, or catalyzed, by a transition metal [2]. Copper is unique in that it allows the use of non-stabilized nucleophiles, such as simple alkyl groups [3-5]. In addition, it is the only metal to enable, at will, con-

\footnotetext{
${ }^{*}$ Correspondence: Prof. Dr. A. Alexakis

Department of Organic Chemistry

University of Geneva

Quai Ernest Ansermet 30

$\mathrm{CH}-1211$ Geneva 4

Tel.: + 41223796522

Fax: + 41223793215
}

E-Mail: alexandre.alexakis@chiorg.unige.ch

http://www.unige.ch/sciences/chiorg/alexakis/group/

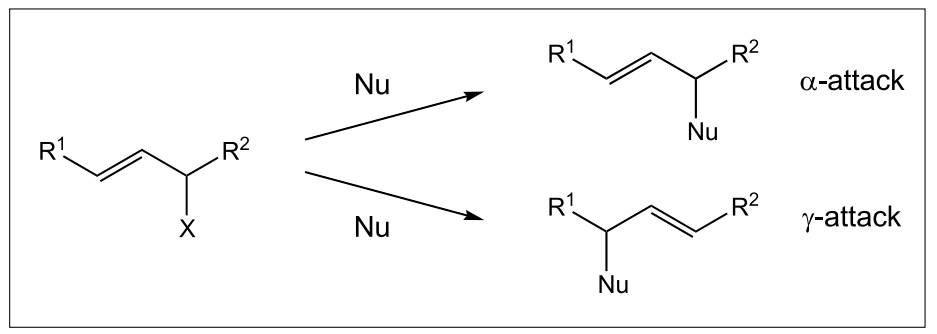

Scheme 1. Regioselectivity of the allylic substitution

trol of the regioselectivity. This control can be easily understood through the usually accepted reaction mechanism (Scheme 2) [6]. Initial formation of a $\pi$-complex [7], is followed by an oxidative addition on the $\gamma$ carbon. The resulting gamma $\sigma$-allyl copper(III) complex may suffer a rapid reductive elimination to afford the $\gamma$-product. If, alternatively, this step is not fast enough, this gamma $\sigma$-allyl copper(III) complex can isomerize into an alpha $\sigma$-allyl copper(III) complex, through a $\pi$-allyl copper(III) complex [8]. Reductive elimination through the less hindered alpha $\sigma$-allyl copper(III) complex will afford the $\alpha$-product. The control of the regioselectivity depends on the nontransferable group $\mathrm{R}^{\mathrm{T}}$. A halide or cyanide group usually allows high $\gamma$ selectivity, whereas an alkyl group (as in $\mathrm{R}_{2} \mathrm{CuLi}$ ) allows an equilibration, thus favoring the substitution at the least sterically hindered carbon [8-14].

With simple substrates, such as the one shown in Scheme 2, $\gamma$-allylation generates a new stereogenic carbon. Therefore, it was tempting to control the reaction to make it enantioselective [15][16]. The first successful attempts were made with a chiral leaving group and a stoichiometric organocopper reagent [17-22]. It was only in 1995 that a catalytic process was disclosed, by Bäckvall and coworkers [23], with a moderate ee of $42 \%$, later improved to $64 \%$ with a new chiral catalyst [24] (Scheme 3). The catalytic system involved a Grignard reagent as primary organometallics, a chiral copper thiolate, and an allylic acetate substituted by an alkyl group. A few years later, in 1999 [25] and 2000 [26], Dubner and Knochel disclosed a different system, based on dialkyl zinc reagents as primary organometallics, an amine as chiral ligand to copper bromide, and an allylic chloride. The results are better when the allylic chloride is substituted by an aryl group, and when the zinc reagent is a hindered one, such as a neopentyl group.

These two catalytic systems are complementary. The dialkylzinc system needs a polar solvent, whereas the Grignard system works better in the least polar solvent. With Grignard reagents, allylic acetates afford 


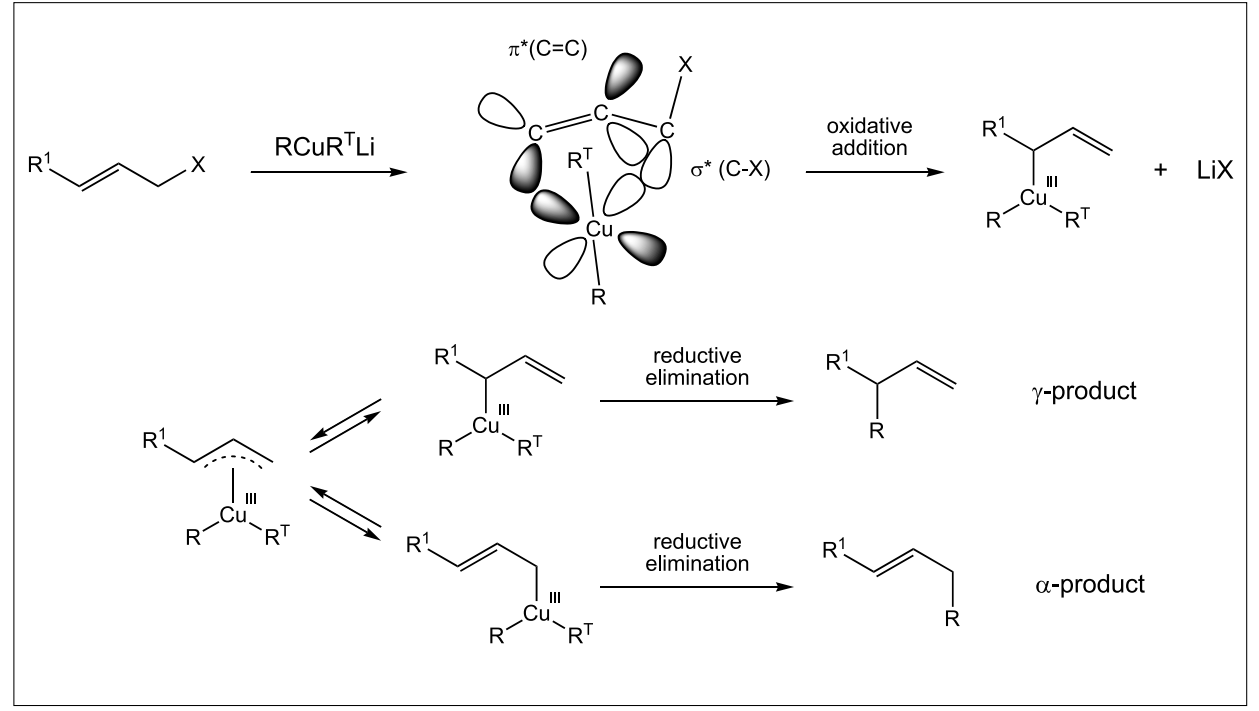

Scheme 2. Mechanism of the Cu-promoted allylic substitution

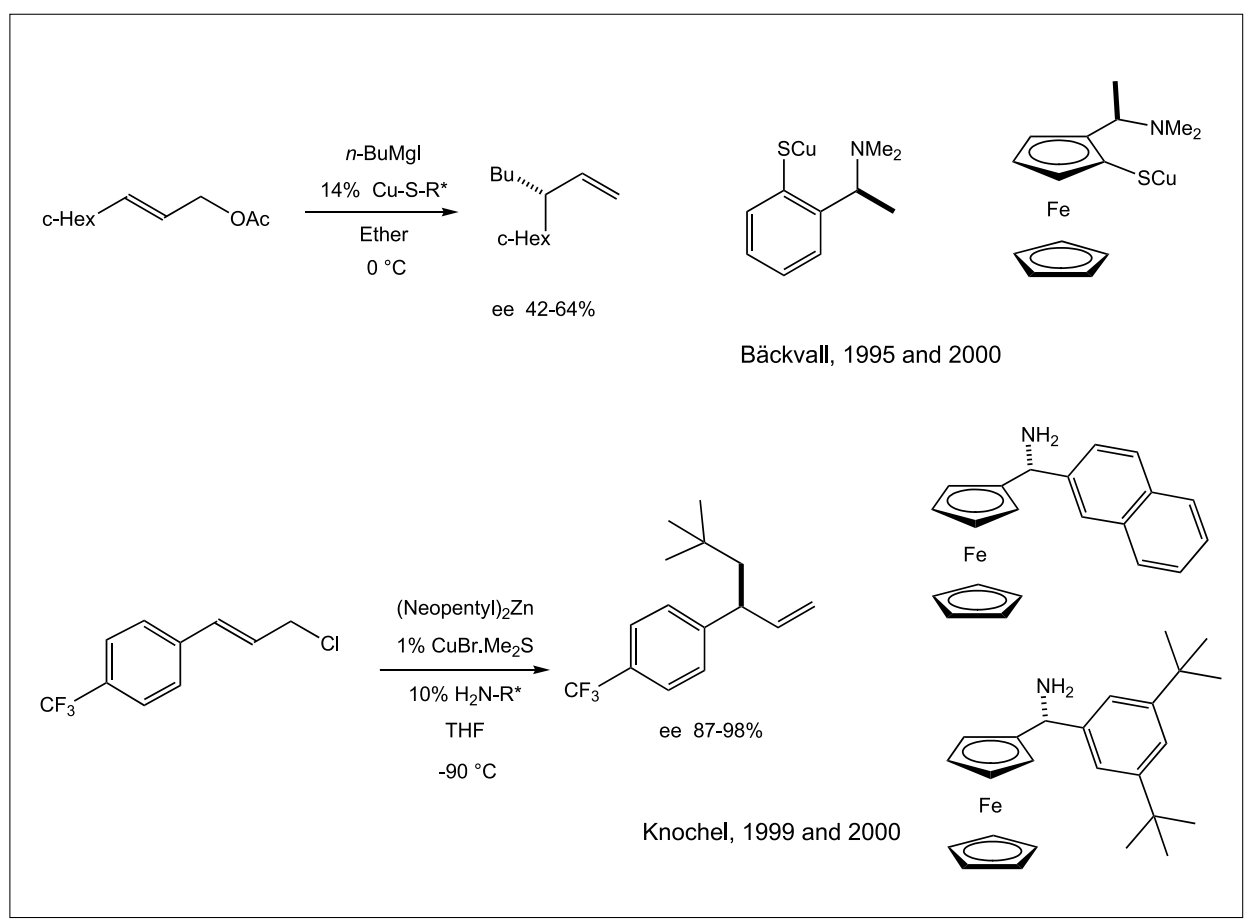

Scheme 3. First copper-catalyzed asymmetric allylic substitution
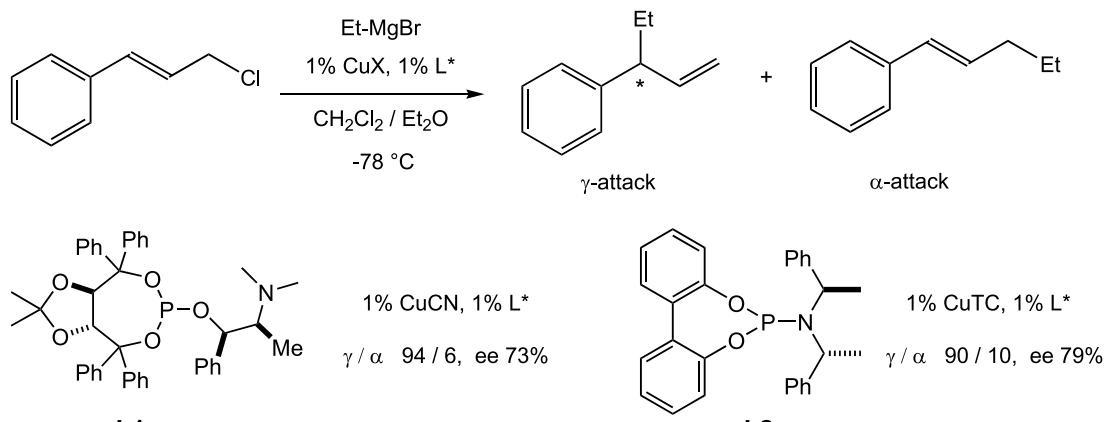

L1

L2 higher stereoselectivity than halides; the reverse is true with dialkylzinc reagents. An alkyl substituent works better than an aryl one on the allylic substrate with Grignards. With dialkylzincs, aryl substituents are preferred; in addition, an electron-withdrawing group affords higher enantioselectivity. With these differences in mind, we shall see how the copper-catalyzed allylic substitution evolved to become presently a very efficient methodology.

\section{Cu-Catalyzed Reactions of Grignard Reagents}

When we started this project, we had first to find the best conditions for an efficient catalytic system in terms of regioselectivity. It appeared that $\mathrm{RMgBr}$ and $\mathrm{RMgCl}$ reagents were better than $\mathrm{RMgI}$, that $\mathrm{CuCN}$ was the best salt (at $1 \%$ loading), that a mixture of $\mathrm{CH}_{2} \mathrm{Cl}_{2}$ and $\mathrm{Et}_{2} \mathrm{O}$ was the best choice of solvent, and that a phosphorous ligand, such as $\mathrm{P}(\mathrm{OEt})_{3}$ had a strong accelerating effect. The screening of three dozens of chiral phosphorus ligands showed that L1 (Scheme 4) was the best one, with 63\% ee and a $\gamma / \alpha$ ratio of $94 / 6$ [27]. The enantioselectivity could be increased to $73 \%$ by slow addition of the Grignard reagent. The best ratio of $\mathrm{Cu}$ to ligand was $1: 1$, at a $1 \%$ catalyst loading. Although $\mathbf{L} 1$ could be considered as a bidentate P,N ligand, we found that phosphoramidite L2, a typical monodentate ligand, could afford similar levels of enantioselectivity [28] (Scheme 4). The major difference of this second generation system was the replacement of $\mathrm{CuCN}$ by $\mathrm{Cu}$-thiophene carboxylate (CuTC) as the copper source. It is striking to note that in the absence of ligand, CuTC gave essentially the $\alpha$-product, whereas $\mathrm{CuCN}$ gave the $\gamma$-product with or without ligand. It may be concluded that the ligand had a strong accelerating effect on the reductive elimination step of the mechanism (Scheme 2).

Ligand L2 was even better than $\mathbf{L 1}$ for the reaction of $i \mathrm{PrMgCl}$, with $83 \%$ ee (instead of 46\%) [28]. The reaction was tested with several cinnamyl-type substrates bearing electron-withdrawing as well as electron-donating groups. In contrast to the dialkylzinc system, which works better with electron-withdrawing groups, they all afford the same level of enantioselectivity (77-86\%) (Scheme 5). In addition, the replacement of phenyl group by an alkyl one (cyclohexyl) is still efficient, with $68 \%$ ee (Scheme 5).

Very recently, a third generation chiral ligand $\mathbf{L 3}$ was disclosed with outstanding results, both in terms of regio- and enantioselectivity [29][30]. It is a bidentate P,O ligand of phosphoramidite type, made of a matched combination of a binaphthol and an amine part (Scheme 6). All the enantiose- 


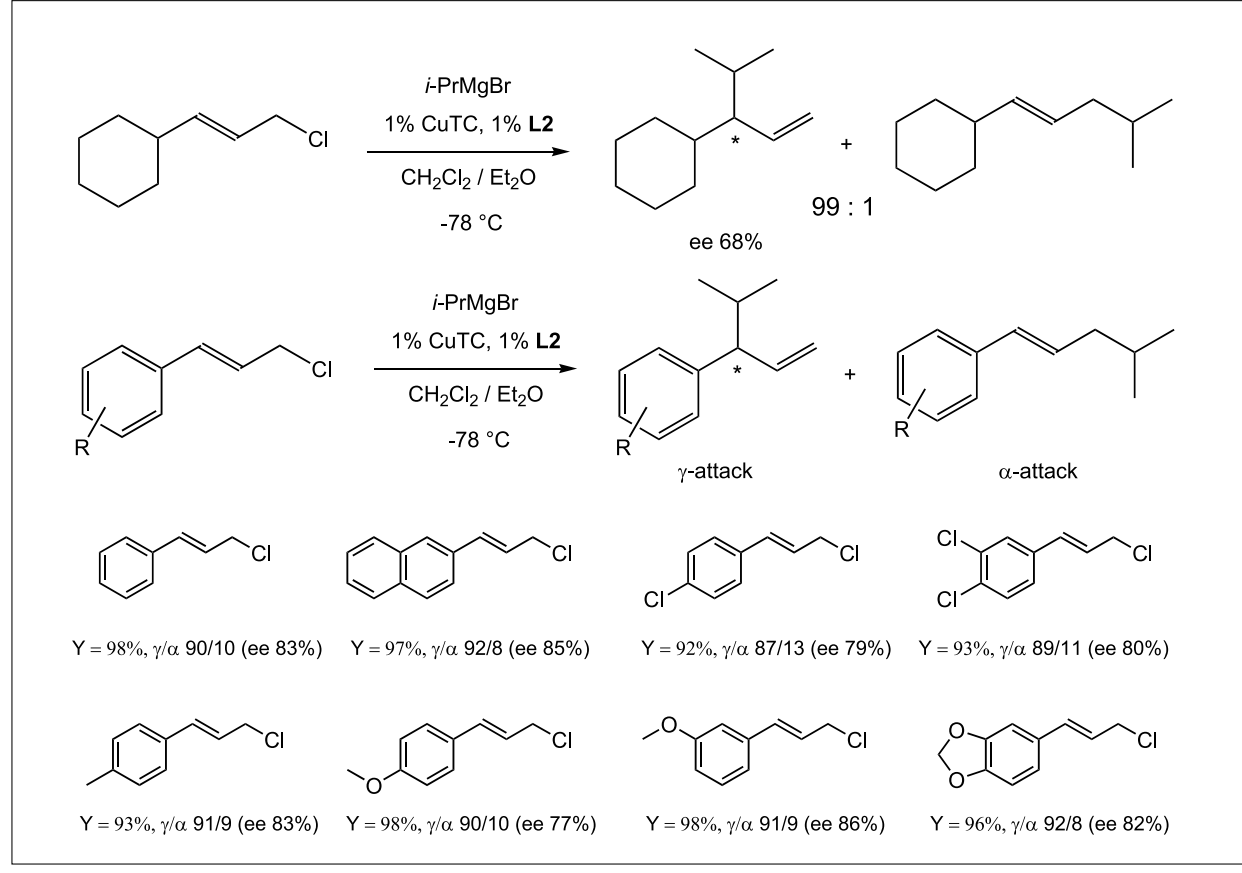

Scheme 5. Reaction of $i \operatorname{PrMgX}$ with alkyl and aryl-substituted allylic chlorides

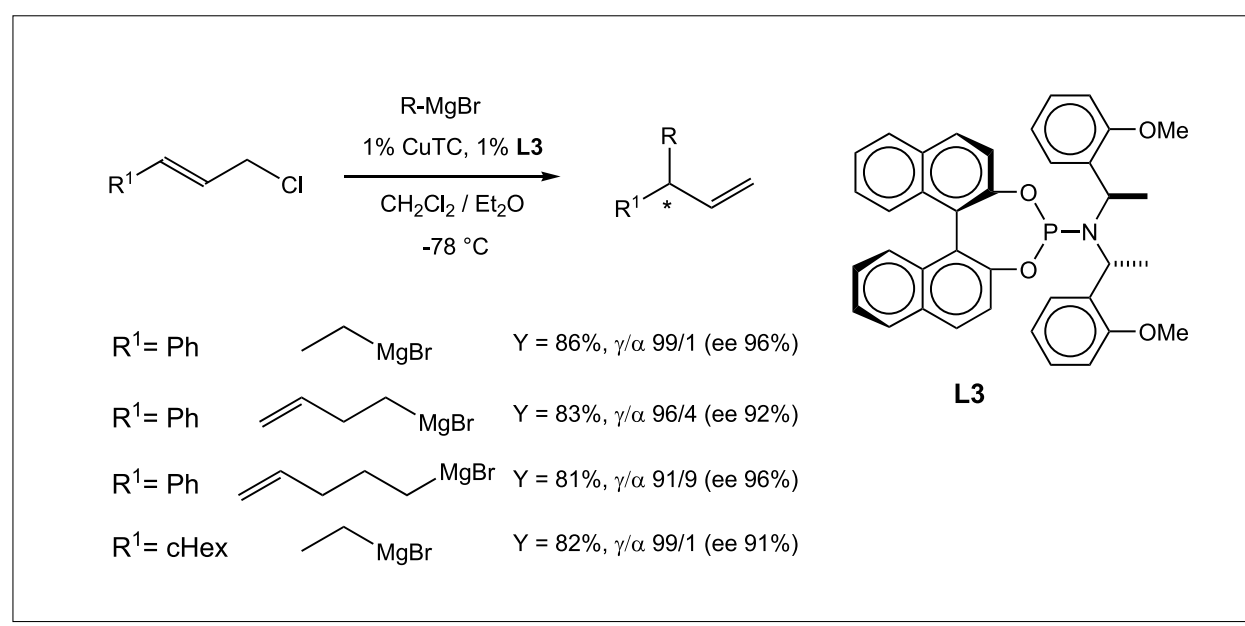

Scheme 6. Third generation ligand for the reaction of alkyl Grignards

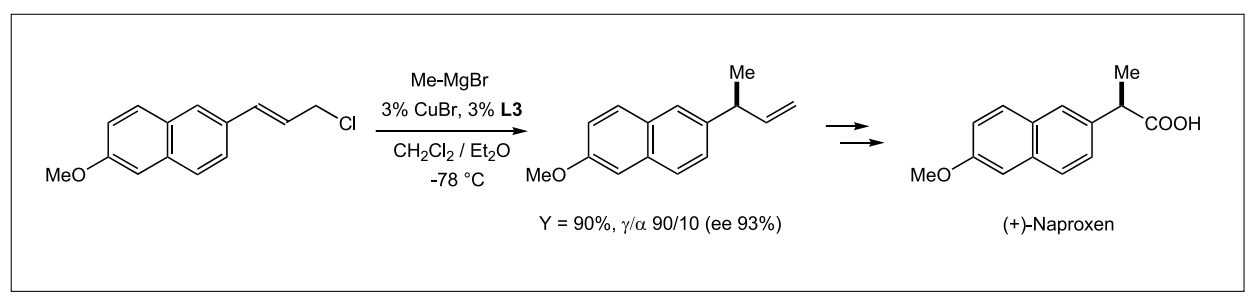

Scheme 7. Synthesis of the precursor of (+)-Naproxen

lectivities are $>91 \%$ and the regioselectivities $>97 / 3$. It works with several Grignard reagents and with variously substituted allylic chlorides. It should also be noted that this ligand also afforded excellent results in the iridium-catalyzed allylic substitution [29][31-33].
$96 \%)$. The reaction was applied to a short synthesis of the precursor of (+)-Naproxen, a well known non-steroidal anti-inflammatory drug (Scheme 7).

The products resulting from the allylic substitution can be transformed into other, more valuable, synthons. Thus, apart from the cleavage of the double bond (as done for (+)-Naproxen), we have studied the possibility offered by the metathesis reaction. Both the cross- and the ring-closing metathesis can be envisioned. It turns out that both reactions are easy to perform, with respectively the second and the first generation Grubbs catalyst [35], and that there is no racemization during that process [28]. Since the metathesis reaction is usually done in $\mathrm{CH}_{2} \mathrm{Cl}_{2}$ solvent, the same as the allylic substitution, it was tempting to see if both reactions can be run in a tandem way, in a one-pot procedure. This is indeed the case, despite the $20 \%$ excess of the Grignard reagent and the copper catalyst. After completion of the allylic substitution, ethyl acrylate could be added, along with the second generation Grubbs catalyst, and after increasing the temperature, the product of the cross metathesis was directly obtained (Scheme 8). Similarly, addition of a Grignard reagent bearing a remote double bond, followed by addition of a first generation Grubbs catalyst, allow the direct obtention ring-closed product [29][30].

Apart from phosphorus ligands, Okamoto and coworkers introduced the use of chiral diaminocarbenes in this reaction [36][37]. The best one bears two 1-naphthyl moieties, and was also very efficient in the conjugate addition reaction [38]. The best substrates were difunctionalized ones, with a $Z$ double bond stereochemistry (Scheme 9 ). It should be pointed out that the $E$ isomer afforded the opposite enantiomer, albeit with a lower enantioselectivity $(60 \%$ instead of $70 \%$ ).

\section{Cu-Catalyzed Reactions of Dialkylzinc Reagents}

In the first reported dialkylzinc system [25][26], the chiral ligand was a chiral amine (at 10\% loading). Alkylzinc halides are not suitable. The best copper salt was $\mathrm{CuBr} . \mathrm{Me}_{2} \mathrm{~S}$, at $1 \%$ loading. Allylic chlorides were the best substrates, and afforded high ee's (44\%-98\%) with the best chiral amine. The best enantioselectivities were obtained with dinoepentylzinc (ee 96\% with cinnamyl chloride), while diethylzinc affords only $44 \%$ ee. An electron-withdrawing group allows an increase of ee to $98 \%$. A cyclohexyl group on the allylic substrate is also possible with a lower ee $(90 \%)$ (Scheme 10).

Chiral amines were also found to be good ligands by Woodward and coworkers 


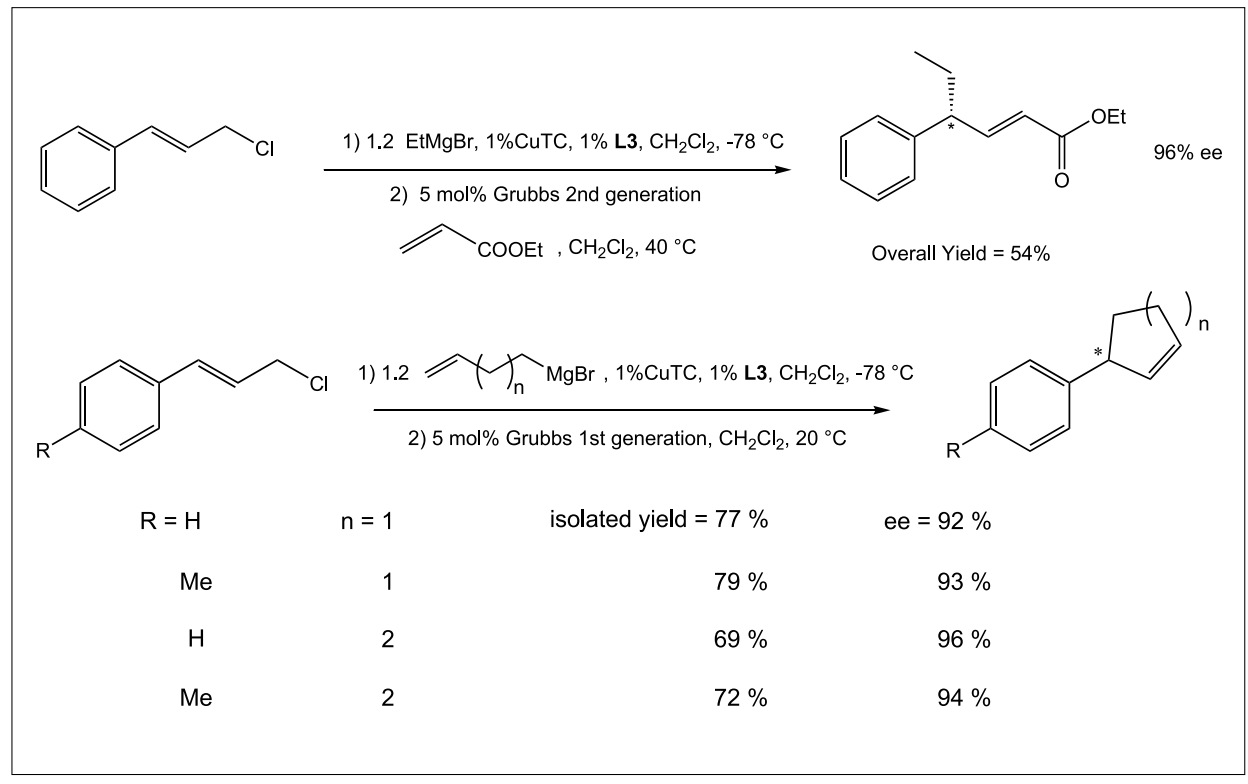

Scheme 8. One-pot allylic substitution/metathesis

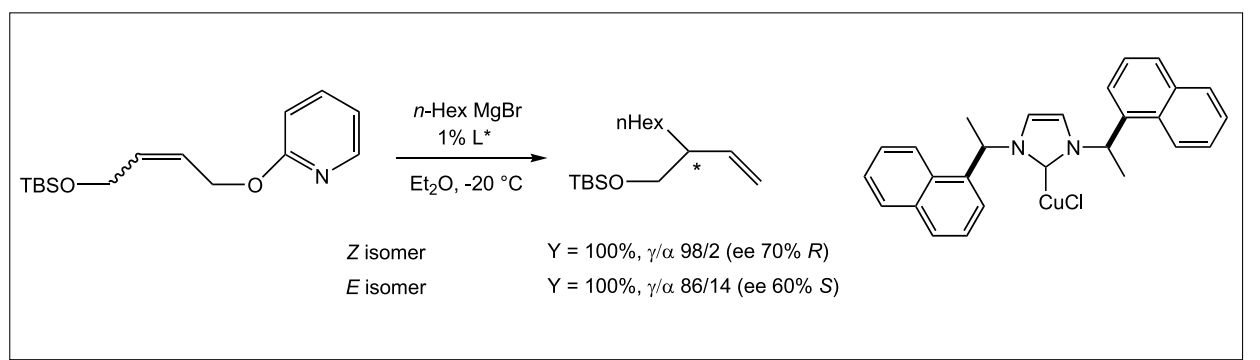

Scheme 9. Copper-carbene complex for the allylic substitution

$$
\begin{aligned}
& \overbrace{\mathrm{Cl}}^{\frac{10 \% \mathrm{~L}^{*}, 1 \% \mathrm{CuBr} \cdot \mathrm{Me}_{2} \mathrm{~S}}{\mathrm{THF},-30^{\circ} \mathrm{C}, 3 \mathrm{~h}}} \\
& \mathrm{R}=\mathrm{Ph} \quad \mathrm{Y}=82 \%, \gamma / \alpha 98 / 2(\text { ee } 96 \%) \\
& p-\mathrm{CF}_{3}-\mathrm{C}_{6} \mathrm{H}_{4} \quad \mathrm{Y}=85 \%, \gamma / \alpha \text { 98/2 (ee 98\%) } \\
& \text { 2-Thienyl } \quad Y=80 \%, \gamma / \alpha 97 / 3 \text { (ee 77\%) } \\
& \text { 1-Naphthyl } \quad Y=72 \%, \gamma / \alpha 98 / 2 \text { (ee 86\%) } \\
& \text { c-Hex } \quad \mathrm{Y}=84 \%, \gamma / \alpha \text { 99/1 (ee } 90 \% \text { ) }
\end{aligned}
$$

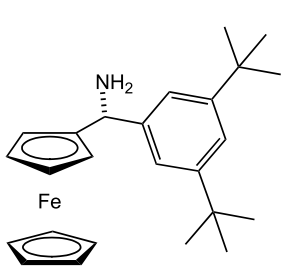

Scheme 10. Allylic substitution with dineopentylzinc<smiles>CC(=O)C(=C[AlH2])CCl</smiles>

\section{$\mathrm{Et}_{2} \mathrm{Zn} / \mathrm{MAO}$}

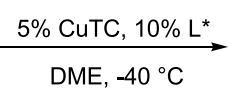<smiles>C=C(C(=O)OC)C(C)C</smiles>

$$
\mathrm{Ar}=\mathrm{Ph}
$$$$
\mathrm{Y}=92 \% \text {, ee } 87 \%
$$$$
p \text {-MeO- } \mathrm{C}_{6} \mathrm{H}_{4}
$$$$
Y=80 \% \text {, ee } 90 \%
$$

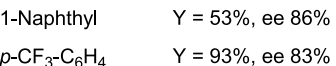$$
p-\mathrm{NO}_{2}-\mathrm{C}_{6} \mathrm{H}_{4} \quad \mathrm{Y}=95 \% \text {, ee } 76 \%
$$

[39]. The Baylis-Hillman derived allylic chloride reacted with diethyl zinc to afford the $\gamma$-product only with moderate enantioselectivities (up to 64\%) with a chiral biphenol ligand [40]. However, during their investigations with phosphoramidite ligands, they found that the true ligand was the chiral amine impurity. Screening of various C2 symmetrical amines allowed them to identify $\mathbf{L} 4$ as the best amine ligand. They also found that the enantioselectivity was higher at the beginning of the reaction. The formation of $\mathrm{ZnCl}_{2}$ during the reaction, shifted the Schlenk equilibrium towards $\mathrm{Et} \mathrm{ZnCl}$, a nonselective reagent. The solution to this problem was to add polymeric methylaluminoxide MAO ([-Al(Me)O $\left.]_{n}\right)$ which shifted back the Schlenk equilibrium to $\mathrm{Et}_{2} \mathrm{Zn}$. Under these conditions they were able to obtain high enantioselectivities (Scheme 11), more so with electron-donating groups than electron-withdrawing ones [39].

Feringa and coworkers used binaphthol-based phosphoramidite ligands. Cinnamyl bromide was the best substrate, the corresponding chloride being too sluggish. The first ligand L5 [41] was later modified to its H8 analog L6 [42] (2\% loading) to improve the results. The reaction is run in THF, with CuOTf $(1 \%)$, and gave better ee's on cinnamyl-type substrates bearing electron-withdrawing substituents (up to $82 \%$ ee). With a cyclohexyl group, the ee drops to $53 \%$ (Scheme 12). Diisopropylzinc reacts faster and affords higher ee $(88 \%)$. In all cases, the regioselectivity remains high. The same reaction was reported by Alexakis and coworkers [29], with the phosphoramidite ligand $\mathbf{L} \mathbf{3}$ which provided higher ee $(91 \%)$. On the other hand, Zhou and coworkers described a new phosphoramidite ligand L7 based on a spiro diol [43]. However, the enantioselectivity was moderate $(71 \%)$ (Scheme 12$)$.

Gennari and coworkers tested a combinatorial library of 125 chiral sulfonamide ligands. Diethylzinc was reacted, in THF, with cinnamyl diethylphosphate, as substrate. However, even with the best copper salt (CuOTf) only 30\% ee was attained [44]. The same screening with a five-membered cyclic meso-type diphosphate was much more successful (Scheme 13). The reaction proceeded in an anti fashion, affording only the $\mathrm{S}_{\mathrm{N}}$, adduct, with high enantioselectivity $(88 \%)$. Dimethylzinc gave even better selectivity (94\% ee), albeit in moderate yield $(40 \%)$. Even phenylation was possible, using a mixture of $\mathrm{Ph}_{2} \mathrm{Zn}$ and $\mathrm{Me}_{2} \mathrm{Zn}$, but with lower ee $(68 \%)$ [45][46]. This is the only report on an efficient transfer of a phenyl group. The reaction with the sixmembered analog was much less selective. Diethyzinc gave only $56 \%$ ee. However, by changing to phosphoramidite ligand $\mathbf{L 5}$, the enantioselectivity jumped to $94 \%$ [46][47]. 


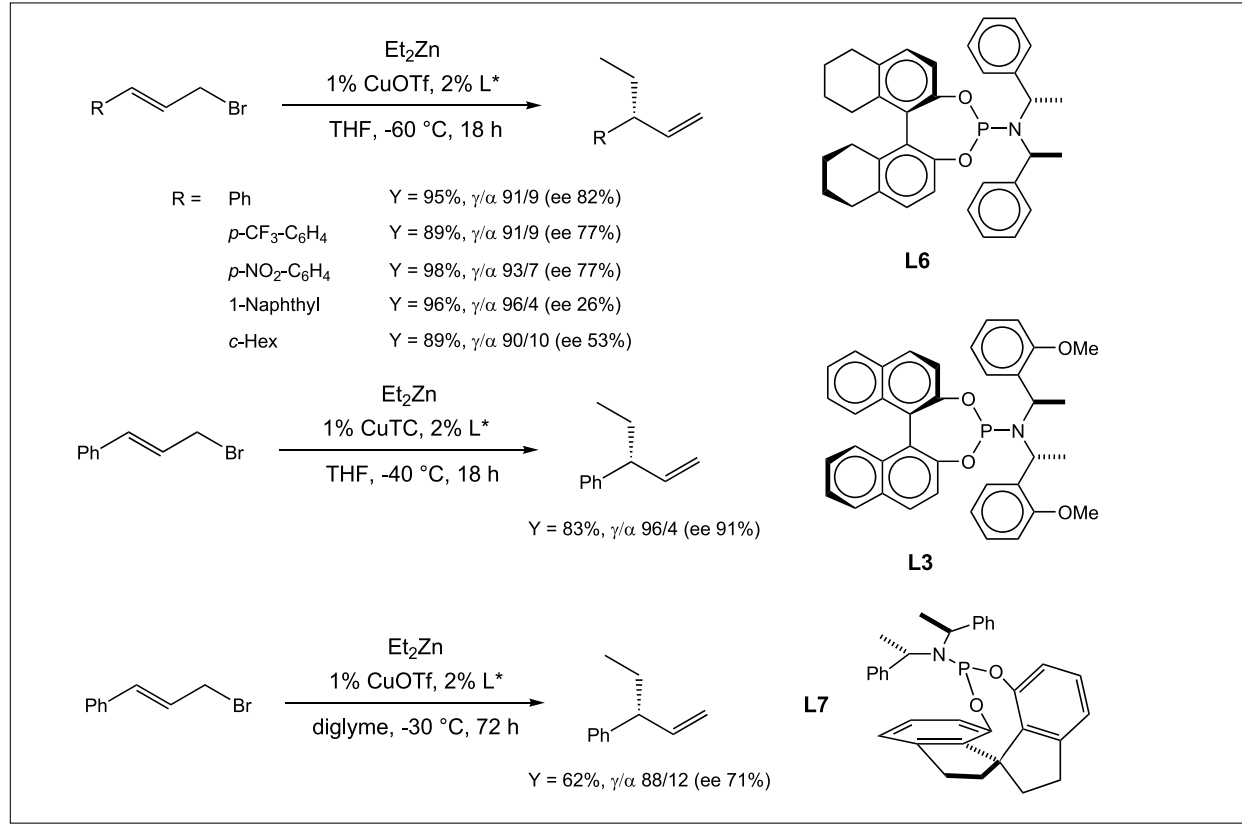

Scheme 12. Allylic substitution with phosphoramidite ligands
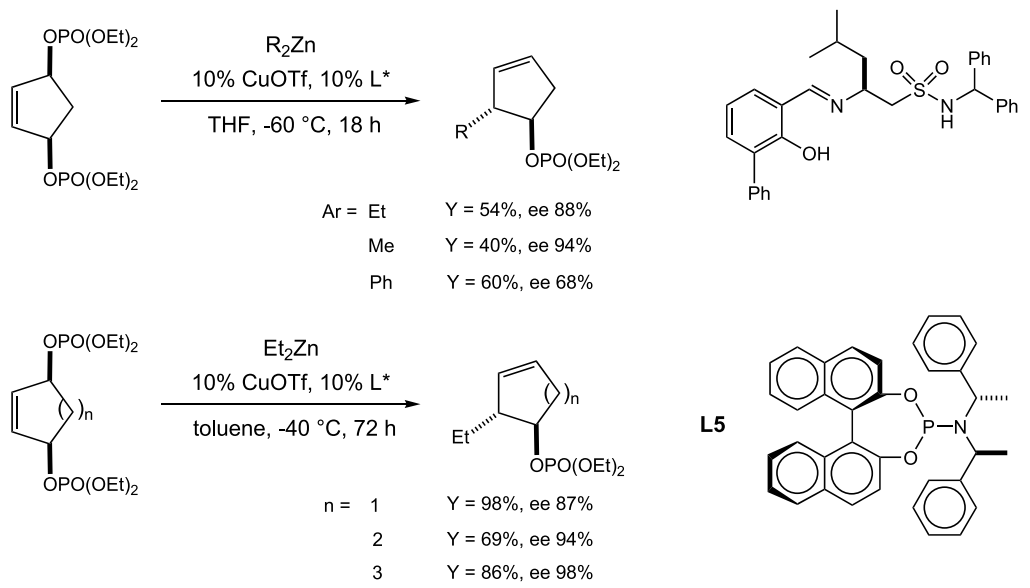

L5

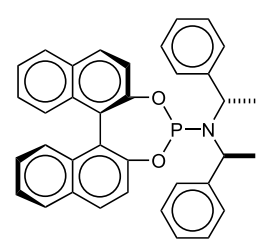

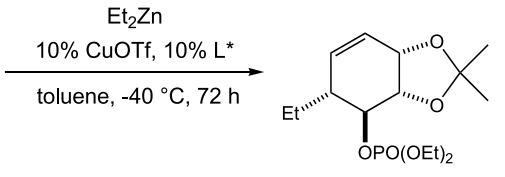

$\mathrm{Y}=52 \%$, ee $86 \%$
The reaction was less anti selective (85/15), but the scope could be extended to sevenmembered ring and more functionalized substrates.

Hoveyda and coworkers also used a combinatorial approach for selecting the most appropriate ligand. These ligands are di- or tripeptides, bearing a hydroxynaphthimine core. They do not incorporate a phosphorus atom (except for the first disclosed ligand). The reaction of dialkylzinc reagent is run at $-15^{\circ}$ to $-50^{\circ} \mathrm{C}$, in THF, with CuOTf (at 10\% loading) and a 1:1 ratio of copper salt to ligand. The allylic substrate has a diethylphosphate as leaving group. Chlorides are not reactive enough or do not deliver high enantioselectivities. Excellent results have been obtained with aryl, alkyl and vinyl substituted allylic systems (ee's 8997\%) [48][49] (Scheme 14). The reaction has also been extended to functionalized substrates, bearing an ester group [50][51]. Dimethylzinc reacts with the same degree of enantioselectivity, but six equivalents are needed to obtain complete conversion. The stereocontrol was suggested to occur on the transition state depicted in Scheme 14 [49]. The phenolic function is acidic enough to be deprotonated by diethylzinc, giving rise to some kind of mixed zinc copper heterocuprate, which also forms a $\pi$-complex with the double bond. On the other hand, zinc is coordinated to the phosphate moiety and the terminal amide of the ligand, thus maintaining the rigidity of the transition state.

One of the most attractive features of this work is the possibility to form chiral quaternary centers by reaction with substrates having a trisubstituted double bond [48-51]. Despite the steric hindrance, excellent levels of regiocontrol could be attained $(>95 \%)$ in most cases. The combinatorial approach of designing ligands allowed Hoveyda and coworkers to find the best suitable ligand for each kind of substrates (Scheme 15).

These methodologies were applied to the short synthesis of natural products (Scheme 16) [48][50][51]. They clearly illustrate the versatility of the copper-catalyzed allylic substitution.

Besides the oligopetide ligands, Hoveyda and coworkers also disclosed some diaminocarbene ligands that efficiently promote the above-mentioned allylic substitutions (Fig.). The first ligand L10 [52] is based on the chirality provided by a binaphthalene core. The X-ray of the carbenecopper complex shows a dimeric structure, and these crystals were catalytically active. Again, a copper phenate is involved. The enantioselectivities are slightly below the best ones shown above, but remain very high ( $>80$ in most cases). Both functionalized and non-functionalized substrates, with di- or trisubstituted double bond, react under similar reaction conditions. Very re- 


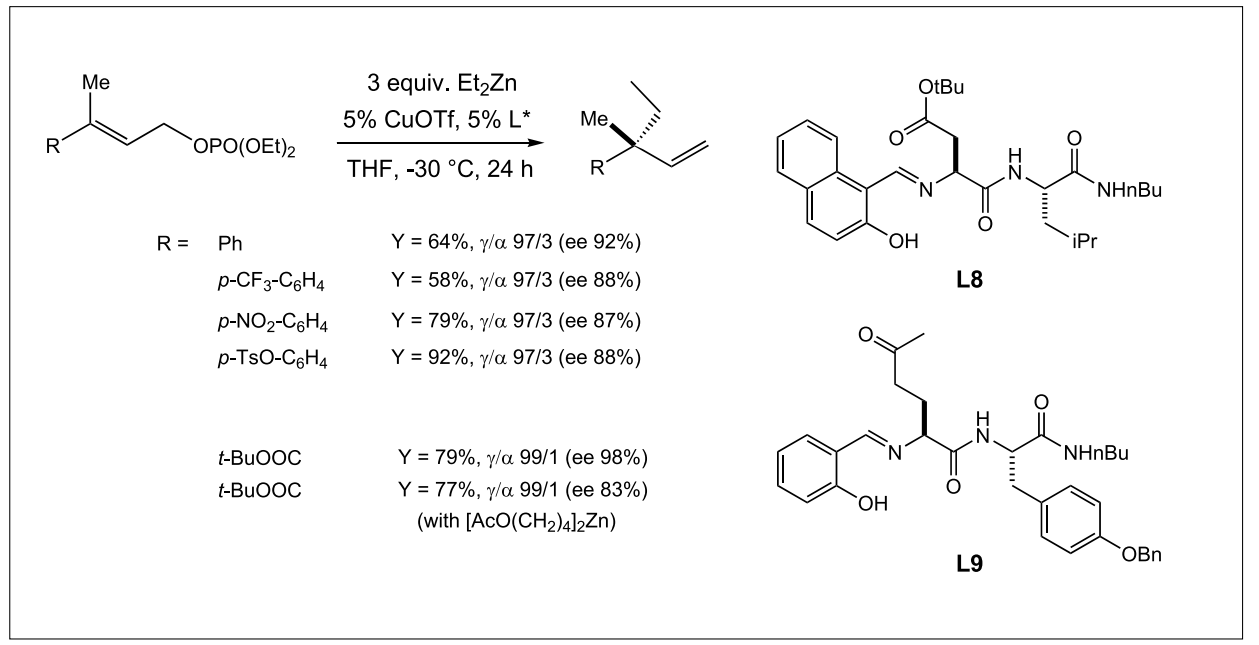

Scheme 15. Allylic substitution on trisubstituted substrates

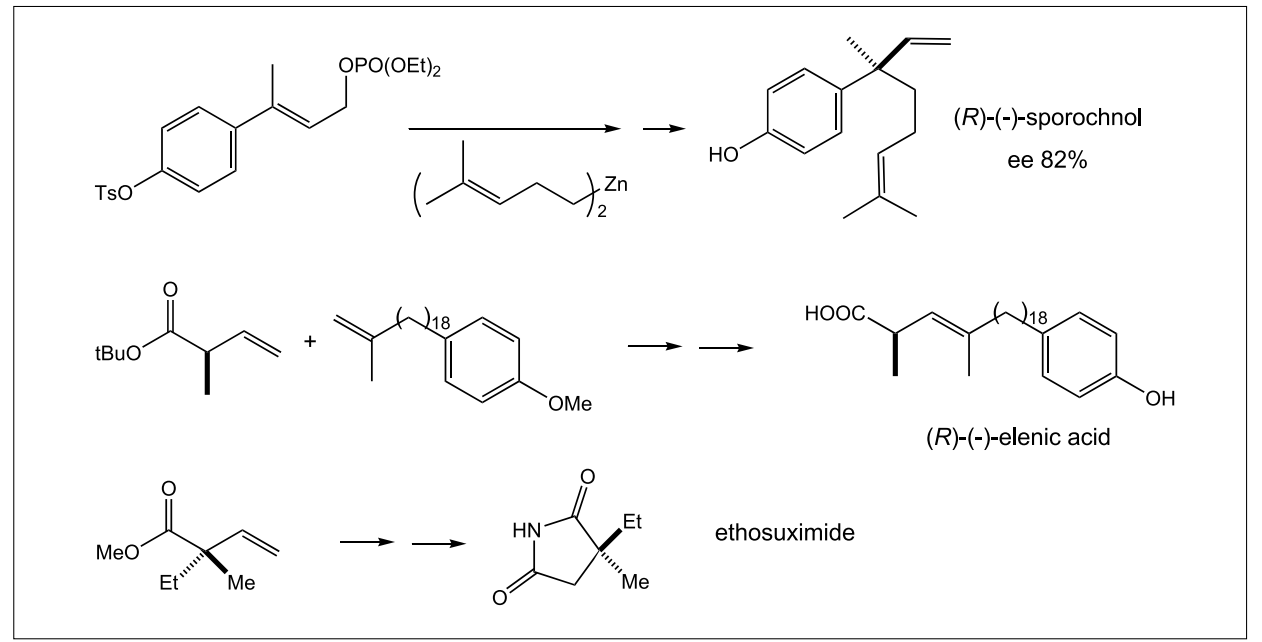

Scheme 16. Applications to the synthesis of natural products
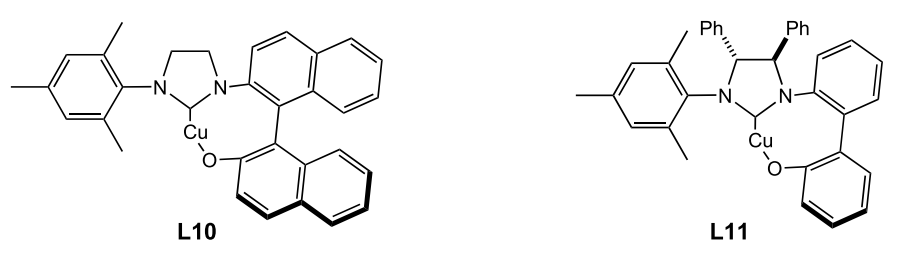

Fig. New carbene ligands for the allylic substitution

cently, a new carbene was disclosed, L11 [53], where the chirality is induced on a flexible biphenyl core, by the chiral backbone. This new ligand provides even better enantioselectivities (>90\%).

\section{Conclusions and Outlook}

This overview of the asymmetric copper-catalyzed allylic substitution shows the rapid growth of this topic. The unique properties of copper, as compared to other tran-
- Besides alkyl groups, is it possible to transfer vinyl, or aryl, or alkynyl groups?

- On the allylic substrate, what other structures are allowed?

- What is the functional compatibility (chemoselectivity) of the reaction?

- Is there a kinetic resolution with chiral substrate?

It is clear that further studies are needed to obtain more information about the reaction mechanism and the enantiodiscriminating steps. And we can be sure that several synthetic applications will soon appear.

Received: January 13, 2006

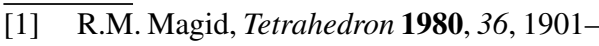
1930.

[2] B.M. Trost, C. Lee, in 'Catalytic Asymmetric Synthesis', 2nd ed., Ed. I. Ojima, Wiley-VCH, New York, 2000, pp. 593-649.

[3] G.H. Posner, Org. Rect. 1975, 22, 253400.

[4] B.H. Lipshutz, S. Sengupta, Org. Rect. 1992, 41, 135-631.

[5] Y.Yamamoto, Angew. Chem. Int. Ed. Engl. 1986, 25, 947-959.

[6] A.S.E. Karlström, J.E. Bäckvall, Chem. Eur. J. 2001, 7, 1981-1989.

[7] E.J. Corey, N.W. Boaz, Tetrahedron Lett. 1984, 25, 3063-3066.

[8] M. Yamanaka, S. Kato, E. Nakamura, $J$. Am. Chem. Soc. 2004, 126, 6287-6293.

[9] J. Levisalles, M. Rudler-Chauvin, H. Rudler, J. Organomet. Chem. 1977, 136, 103-110.

[10] J.P. Marino, D.M. Floyd, Tetrahedron Lett. 1979, 25, 675-679.

[11] C.C. Tseng, S.D. Paisley, H.L. Goering, J. Org. Chem. 1986, 51, 2884-2891.

[12] C.C. Tseng, S.J. Yen, H.L. Goering, J. Org. Chem. 1986, 51, 2892-2895.

[13] J.E. Bäckvall, M. Sellén, B. Grant, J. Am. Chem. Soc. 1990, 112, 6615-6621.

[14] J.E. Bäckvall, E.S.M. Persson, A. Bombrun, J. Org. Chem. 1994, 59, 4126-4130.

[15] A.S.E. Karlström, J.E. Bäckvall, Modern Organocopper Chemistry, Ed. N. Krause, Wiley-VCH, Weinheim, 2001, 259-288.

[16] H. Yorimitsu, K. Oshima, Angew. Chem. Int. Ed. 2005, 44, 4435-4439.

[17] A. Alexakis, P. Mangeney, A. Ghribi, I. Marek, R. Sedrani, C. Guir, J.F. Normant, Pure and Appl. Chem. 1988, 60, 49-56.

[18] S.E. Denmark, L.K. Marble, J. Org. Chem. 1990, 55, 1984-1986.

[19] V. Caló, V. Fiandanese, A. Nacci, A. Scilimati, Tetrahedron 1994, 50, 7283-7292.

[20] V. Caló, A. Nacci, V. Fiandanese, Tetrahedron 1996, 52, 10799-10810.

[21] H.-J. Gais, H. Müller, J. Bund, M. Scommoda, J. Brandt, G.J. Raabe, Am. Chem. Soc. 1995, 117, 2453-2466.

[22] B. Breit, D. Breuninger, Synthesis 2005, 147-157.

[23] M. van Klaveren, E.S.M. Persson, A. del Villar, D.M. Grove, J.E. Bäckvall, G. van 
Koten, Tetrahedron Lett. 1995, 36, 30593062.

[24] A.S.E. Karlstrom, F.F. Huerta, G.J. Meuzelaar, J.E. Bäckvall, Synlett 2001, 923926.

[25] F. Dubner, P. Knochel, Angew. Chem. Int. Ed. 1999, 38, 379-381.

[26] F. Dubner, P. Knochel, Tetrahedron Lett. 2000, 41, 9233-9237.

[27] A. Alexakis, C. Malan, L. Lea, C. Benhaim, X. Fournioux, Synlett 2001, 927930.

[28] A. Alexakis, K. Croset, Org. Lett. 2002, 4, 4147-4149.

[29] K. Tissot-Croset, D. Polet, A. Alexakis, Angew. Chem. Int. Ed. 2004, 43, 24262428.

[30] K. Tissot-Croset, D. Polet, S. Gille, C. Hawner, A. Alexakis, Synthesis 2004, 2586-2590.

[31] A. Alexakis, D. Polet, Org. Lett. 2004, 6, 3529-3532.

[32] C. Welter, A. Dahnz, B. Brunner, S. Streiff, P. Dübon, G. Helmchen, Org. Lett. 2005, 7, 1239-1242; S. Streiff, C. Welter, M. Schelwies, G. Lipkowsky, N. Miller, G. Helmchen, Chem. Comm. 2005, 29572959.

[33] R. Weihofen, A. Dahnz, O. Tverskoy, G. Helmchen, Chem. Comm. 2005, 35413543.

[34] K. Tissot-Croset, A. Alexakis, Tetrahedron Lett. 2004, 45, 7375-7378.

[35] T.M. Trnka, R.H. Grubbs, Acc. Chem. Res. 2001, 34, 18.

[36] S. Tominaga, Y. Oi, T. Kato, D.K. An, S. Okamoto, Tetrahedron Lett. 2004, 45, 5585-5588.

[37] S. Okamoto, S. Tominaga, N. Saimo, K. Kase, K. Shomoda, J. Organomet. Chem. 2005, 690, 6001-6007.

[38] A. Alexakis, C.L. Winn, F. Guillen, J. Pitkowicz, S. Roland, P. Mangeney, Adv. Synth \& Cat. 2003, 345, 345-348.
[39] P.J. Goldsmith, S.J. Teat, S. Woodward, Angew. Chem. Int. Ed. 2005, 44, 22352237.

[40] C. Börner, J. Gimeno, S. Gladiali, P.J. Goldsmith, D. Ramazzotti, S. Woodward, Chem. Comm. 2005, 3541-3543.

[41] H. Malda, A.W. van Zijl, L.A. Arnold, B.L. Feringa, Org. Lett. 2001, 3, 1169-1171.

[42] A.W. van Zijl, L.A. Arnold, A.J. Minaard, B.L. Feringa, Adv. Synth \& Cat. 2004, 346, 413-420.

[43] W.J. Shi, L.X. Wang, Y. Fu, S.F. Zhu, Q.L. Zhou, Tetrahedron: Asymmetry 2003, 14 , 3867-3872.

[44] S. Ongeri, U. Piarulli, M. Roux, C. Monti, C. Gennari, Helv. Chim. Acta 2002, 85, 3388-3399.

[45] U. Piarulli, P. Daubos, C. Claverie, M. Roux, C. Gennari, Angew. Chem. Int. Ed. 2003, 42, 234-236.

[46] U. Piarulli, P. Daubos, C. Claverie, C. Monti, C. Gennari, Eur. J. Org. Chem. 2005, 895-906.

[47] U. Piarulli, C. Claverie, P. Daubos, C. Gennari, A.J. Minaard, B.L. Feringa, Org. Lett. 2003, 5, 4493-4496.

[48] C.A. Luchaco-Cullis, H. Mizutani, K.E Murphy, A.H. Hoveyda, Angew. Chem. Int. Ed. 2001, 40, 1456-1460.

[49] M.A. Kacprzynski, A.H. Hoveyda, J. Am. Chem. Soc. 2004, 126, 10676-10681.

[50] K.E. Murphy, A.H. Hoveyda, J. Am. Chem. Soc. 2003, 125, 4690-4691.

[51] K.E. Murphy, A.H. Hoveyda, Org. Lett. 2005, 7, 1255-1258.

[52] A.O. Larsen, W. Leu, C. Nieto Oberhuber, J.E. Campbell, A.H. Hoveyda, J. Am. Chem. Soc. 2004, 126, 11130-11131.

[53] J.J. van Veldhuisen, J.E. Campbell, R.E. Guidici, A.H. Hoveyda, J. Am. Chem. Soc. 2005, 127, 6877-6882. 